\title{
A Real-Time Cross-modality Correlation Filtering Method for Referring Expression Comprehension
}

\author{
Yue Liao ${ }^{1,3} \mathrm{Si} \mathrm{Liu}^{1 *}$ Guanbin $\mathrm{Li}^{2}$ Fei Wang ${ }^{3}$ Yanjie Chen ${ }^{3}$ Chen Qian ${ }^{3}$ Bo Li ${ }^{1}$ \\ ${ }^{1}$ School of Computer Science and Engineering, Beihang University \\ ${ }^{2}$ Sun Yat-sen University ${ }^{3}$ SenseTime Research \\ liaoyue.ai@gmail.com; \{liusi, boli\}@buaa.edu.cn; liguanbin@mail.sysu.edu.cn; \\ \{wangfei, chenyanjie, qianchen\}esensetime.com
}

\begin{abstract}
Referring expression comprehension aims to localize the object instance described by a natural language expression. Current referring expression methods have achieved good performance. However, none of them is able to achieve realtime inference without accuracy drop. The reason for the relatively slow inference speed is that these methods artificially split the referring expression comprehension into two sequential stages including proposal generation and proposal ranking. It does not exactly conform to the habit of human cognition. To this end, we propose a novel Realtime Cross-modality Correlation Filtering method (RCCF). $R C C F$ reformulates the referring expression comprehension as a correlation filtering process. The expression is first mapped from the language domain to the visual domain and then treated as a template (kernel) to perform correlation filtering on the image feature map. The peak value in the correlation heatmap indicates the center points of the target box. In addition, RCCF also regresses a 2-D object size and 2-D offset. The center point coordinates, object size and center point offset together to form the target bounding box. Our method runs at 40 FPS while achieving leading performance in RefClef, RefCOCO, RefCOCO+ and RefCOCOg benchmarks. In the challenging RefClef dataset, our methods almost double the state-of-the-art performance (34.70\% increased to $63.79 \%)$. We hope this work can arouse more attention and studies to the new cross-modality correlation filtering framework as well as the one-stage framework for referring expression comprehension.
\end{abstract}

\section{Introduction}

Referring expression comprehension [34, 32, 27] has attracted much attention in recent years. A referring expression is a natural language description of a particular object

\footnotetext{
${ }^{*}$ Corresponding author
}

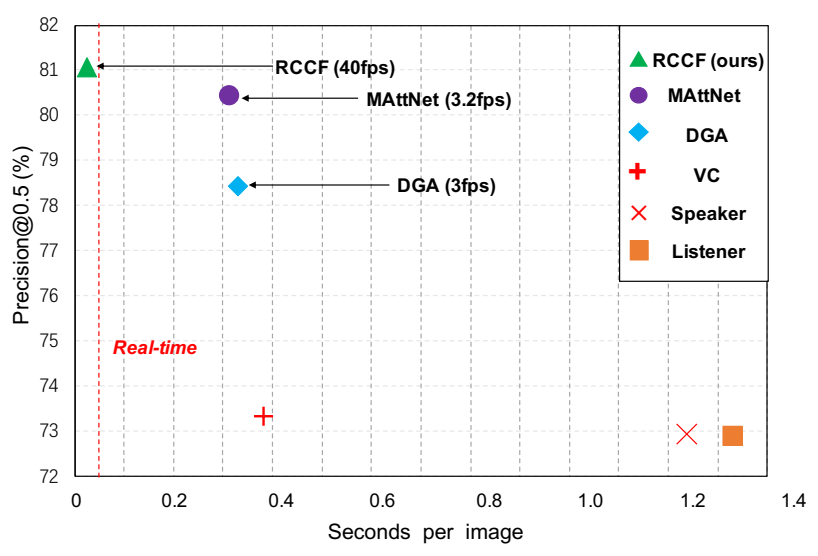

Figure 1. Precision (IOU $>0.5)$ versus inference time on the RefCOCO testA set at single Titan Xp GPU. Our method RCCF achieves $40 \mathrm{fps}(0.25 \mathrm{~ms}$ per image $)$, which exceeds the real-time speed of $25 \mathrm{fps}$ and is significantly faster than existing methods by a significant margin (12 times). The precision of RCCF also outperforms the state-of-the-art methods.

in an image. Given such a referring expression, the target of referring expression comprehension is to localize the object instance in the image. It is one of the key tasks in the field of machine intelligence to realize human-computer interaction, robotics and early education.

Conventional methods for referring expression comprehension mostly formulate this problem as an object retrieval task, where an object that best matches the referring expression is retrieved from a set of object proposals. These methods [32, 29, 28, 27] are mainly composed of two stages. In the first stage, given an input image, a pre-trained object detection network is applied to generate a set of object proposals. In the second stage, given an input expression, the best matching region from the detected object proposals is selected. Although existing two-stage methods have achieved great advance, there are still some problems. 1) The performance of the two-stage methods is very limited to the qual- 
ity of object proposals generated in the first stage. If the target object is not accurately detected, it is impossible to match the language in the second stage. 2) In the first stage, a lot of extra object detection data, i.e., COCO [17] and Visual Genome [13], are indispensable to achieve satisfactory result. 3) Two-stage methods are usually computationally costly. For each object proposal, both feature extraction and cross-modality similarity computation should be conducted. However, only the proposal with highest similarity is selected finally. As we can see in Figure 1, the accuracy of current two-stage methods is reasonable while the inference speed still has a large gap to reach real-time.

The three aforementioned problems are difficult to solve in existing two-stage frameworks. We reformulate referring expression comprehension as a cross-modality template matching problem, where the language serves as the template(filter kernel) and the image feature map is the search space to perform correlation filtering on. Mathematically, referring expression comprehension aims to learn a function $f(z, x)$ that compares an expression $z$ to a candidate image $x$ and returns a high score in the corresponding regions. The region is represented by 2-dim center point, 2-dim object size (height and width) and 2-dim offset to recover the discretization error [15, 36, 6]. Our proposed RCCF is end-to-end trainable. The language embedding is used as correlation filter and applied to the feature map to produce the heatmap for center point. For more accurate localization, we compute the correlation map on multi-level image feature and fuse the output maps to produce the final heatmap of object center. Moreover, the width, height and offset heatmap are regressed with visual feature only. During inference, the text is first embedded into visual space and then slides on the image feature maps. The peak point in the object center heatmap is selected as the center of the target. The corresponding width, height and offset are collected to form the target bounding box, which is the referring expression comprehension result.

The advantages of our proposed RCCF method can be summarized as three-folds:

- The inference speed of our method reaches real-time (40 FPS) with a single GPU, which is 12-times faster than the two-stage methods.

- Our method can be trained with referring expression dataset only, with no need for any additional object detection data. Moreover, our one-stage model can avoid error accumulation from the object detector in traditional two-stage methods.

- RCCF has achieved the state-of-the-art performance in RefClef, RefCOCO, RefCOCO+ and RefCOCOg datasets. Especially, in the RefClef dataset, our method outperforms the state-of-the-art methods by a significant margin from $34.70 \%$ to $63.79 \%$, almost double the performance of the state-of-the-art method.

\section{Related Work}

\subsection{Referring Expression Comprehension}

Conventional methods for referring expression comprehension are mostly composed of two-stage. In the first stage, given an input image, a pre-trained object detection network or an unsupervised method is applied to generate a set of object proposals. In the second stage, given an input expression, the best matching region is selected from the detected object proposals. With the development of deep learning, the two-stage methods has achieved great progress. The most two-stage methods focus on improving the second stage. Most of them [20, 9, 35, 32, 27, 28] mainly focus on exploring how to mine context information from the language and image or model the relationship between referents, for example, MAttNet [32] proposed a modular attention model to capture multi-modality context information.

Though existing two-stage methods have achieved pretty-well performance, there are some common problems. Firstly, the performance of two-stage methods is limited to the object detectors. Secondly, these methods waste a lot of time in object proposals generation and features extraction for each proposal. Therefore, we propose to localize the target object directly given an expression with our correlation filtering based method.

\subsection{Correlation Filtering}

The correlation filtering is firstly proposed to train a linear template to discriminate between images and their translations. The correlation filtering is widely used in different areas of computer vision. Object classification [14, 7, 26] can be seen as a correlation filtering task, where the output image feature vector can be seen as a filter kernel, which performs correlation filtering on the weight matrix of the last multi-layer perceptron. For single object tracking, which aims to localize an object in a video given the object region in the first frame, the correlation filtering can play a role in comparing the first frame with the rest ones. The early works [2, 8] in tracking firstly transfer the image into Fourier domain, and perform correlation filtering in Fourier domain. Siamese FC [1] proposed to directly learn a correlation layer on the spatial domain, where Siamese FC compares two image features extracted from a Siamese network.

Inspired by human visual perception mechanism, we believe that the process of performing language based visual grounding can be analogized to the process of filter-based visual response activation. Specifically, people generally comprehend the semantic information of a sentence in a 
global way, and form a feature template about the sentence description in the mind, then quickly perform attention matching on the image based on the template, wherein the salient region with the highest response value is considered as the target matching region. To this end, we formulate the problem of referring expression comprehension as a cross-modality correlation filtering process and solve with a single-stage joint optimization paradigm.

\section{Method}

In this section, we introduce our proposed RCCF method for referring expression comprehension. Our goal is to localize the object described by the reference expression directly without proposal generation step. To this end, we formulate referring expression comprehension task as a crossmodality template matching problem. In RCCF, we first localize the center point of the object described by the expression by performing correlation filtering on the image feature with a language-guided filter kernel. Then, we apply a regression module to regress the object size and center point offset. The peak value in the correlation heatmap, the regressed object size and center point offset together form the target bounding box.

\subsection{Framework}

Let $Q$ represent a query sentence and $I \in \mathbb{R}^{H \times W \times 3}$ denote the image of width $W$ and height $H$. Our aim is to find the object region described by the expression. The target object region is represented by its center point $\left(x_{t}, y_{t}\right)$ and the object size $\left(w_{t}, h_{t}\right)$. Additionally, to recover the discretization error caused by the output stride, we predict a local offset $\left(\delta x_{t}, \delta y_{t}\right)$ for the center point $t$. To sum up, the referring expression comprehension can be formulated as a mapping function $\left(x_{t}, y_{t}, w_{t}, h_{t}, \delta x_{t}, \delta y_{t}\right)=\phi(Q, I)$.

As shown in Figure 2, our proposed RCCF is composed of three modules, i.e., expression and image encoder, correlation filtering as well as size and offset regression modules. The expression and image encoder module includes the language feature extractor $L(\cdot)$ and visual feature extractor $E(\cdot)$. The extracted features are represented as $L_{Q}$ and $E_{I}$ respectively. The expression feature $L_{Q}$ is then mapped from the language domain to the visual domain by the cross-modality mapping function $M(\cdot)$. The correlation filtering module treats the mapping result $M\left(L_{Q}\right)$ as the filter (kernel) to convolve with the visual feature map $E_{I}$ and produces a heatmap $C \in \mathbb{R}^{\frac{H}{d} \times \frac{W}{d}}$, where $d$ is the output stride. The peak value of $C$ indicates the center point of the object $(x, y)$ depicted by the expression. Moreover, the size and offset regression module predicts the object size $(w, h)$ and local offset of the center point $(\delta x, \delta y)$. Next, we will introduce the three modules in detail.

\subsection{Expression and Image Encoder}

The expression encoder $L(\cdot)$ takes the expression as input, and produces a 512-D feature vector. We first embed the expression into a 1024-D vector, followed by a fully connected layer to transform the vector into 512-D. Then we feed the transformed feature into a Bi-LSTM to get the expression feature $L_{Q}$.

The image encoder $E(\cdot)$ adopts the Deep Layer Aggregation (DLA) [31] architecture with deformable convolution [4]. DLA is an image classification network with hierarchical skip connections. Following Centernet [36], we use the modified DLA network with 34 layers, which replace the skip connection with the deformable convolution. Because a referring expression may consist of various kinds of semantic information such as attribute, relationship and spatial location. To well match the expression, we use three level visual features. As shown in Figure 2, we extract three level features $\left[E_{I}^{1}, E_{I}^{2}, E_{I}^{3}\right]=E(I)$ from the DLA net which are transformed into a unified size $\frac{H}{d} \times \frac{W}{d}$ from $\frac{H}{8 d} \times \frac{W}{8 d}, \frac{H}{4 d} \times \frac{W}{4 d}$, and $\frac{H}{2 d} \times \frac{W}{2 d}$ respectively. The size of $\left[E_{I}^{1}, E_{I}^{2}, E_{I}^{3}\right]$ are all $64 \times \frac{H}{d} \times \frac{W}{d}$. When computing the correlation map $\hat{C}$, all three level features are utilized. During regression process, only $E_{I}^{1}$ with the highest resolution is used for computational efficiency.

\subsection{Cross-modality Correlation Filtering}

The aim of cross-modality correlation filtering is to localize the center of the target box $(x, y)$. It contains three steps, including language-guided kernel generation, cross-modality correlation operation and correlation maps fusion. Firstly, we utilize three different linear functions to generate three filter kernels $\left[k_{1}, k_{2}, k_{3}\right]=$ $\left[M_{1}\left(L_{Q}\right), M_{2}\left(L_{Q}\right), M_{3}\left(L_{Q}\right)\right]$ from the expression feature $L_{Q}$. The three fully connected layers $M_{1}(\cdot), M_{2}(\cdot)$ and $M_{3}(\cdot)$ serve as the cross-modality mapping function to project from the expression space to the visual space. Each kernel is a 64-D feature vector which is then reshaped into a $64 \times 1 \times 1$ filter for subsequent operations. Secondly, we perform correlation operation on the three levels of visual features with their corresponding language-mapped kernels $\left[C^{1}, C^{2}, C^{3}\right]=\left[k_{1} * E_{I}^{1}, k_{2} * E_{I}^{2}, k_{3} * E_{I}^{3}\right]$, where $*$ denotes convolution operation. Thirdly, the three correlation maps are pixel-wisely averaged and fed into an activation function $\hat{C}=\operatorname{Sigmod}\left(\frac{C^{1}+C^{2}+C^{3}}{3}\right)$. The size of $\hat{C}, C^{1}, C^{2}$ and $C^{3}$ are all $\mathbb{R}^{\frac{H}{d} \times \frac{W}{d}}$. The location with highest score in $\hat{C}$ is the center point of the target object.

We train the center point prediction network following [15, 36]. For the ground-truth center point $\left(\tilde{x}^{g}, \tilde{y}^{g}\right)$, we compute a low-resolution equivalent $\left(x^{g}, y^{g}\right)=\left\lfloor\frac{\left(\tilde{x}^{g}, \tilde{y}^{g}\right)}{d}\right\rfloor$ by considering the output stride $d$. We use the Gaussian kernel $C_{x y}=\exp \left(-\frac{\left(x-x^{g}\right)^{2}+\left(y-y^{g}\right)^{2}}{2 \sigma_{t}^{2}}\right)$ to splat the ground- 


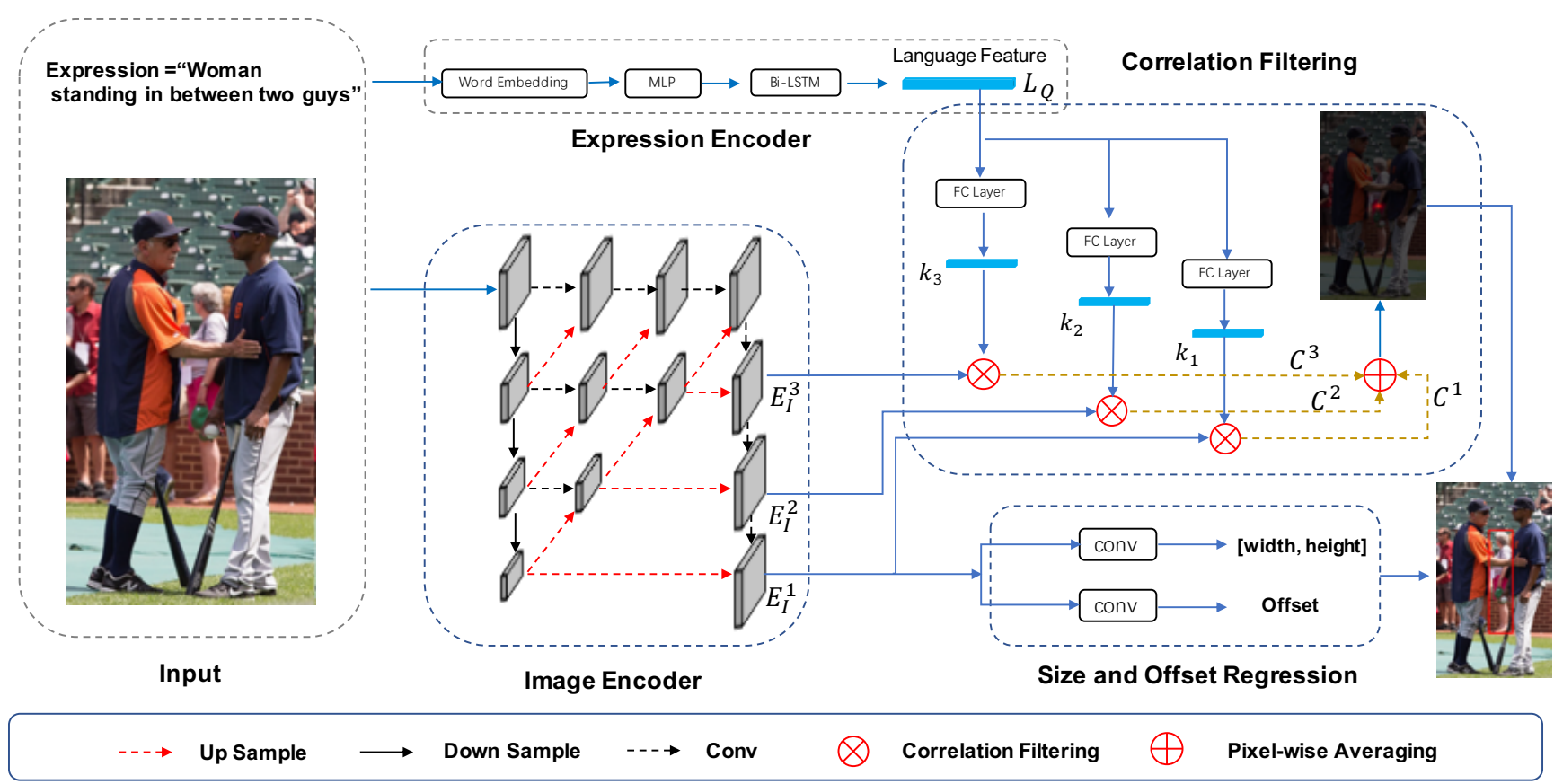

Figure 2. Overview of the proposed RCCF framework. a) Expression and Image Encoder: Bi-LSTM and DLA structure are used for expression and visual feature extraction. b) Cross-modality Correlation Filtering: the extracted language feature is mapped into three different filter kernels. Then we perform correlation filtering on three levels of image features with the corresponding kernel to generate three correlation maps respectively. Finally, we fuse the three correlation maps by pixel-wise averaging. The center point corresponds to the peak value of the fused heatmap. c) Size and Offset Regression: the 2-dim object size and the local offset for the center point are regressed based on the last-level image feature only. The target object region is obtained by combining the estimated center point, the object size and the local offset.

truth center point in a heatmap $C \in[0,1]^{\frac{W}{d} \times \frac{H}{d}}$, where $C_{x y}$ is the value of $C$ at the spatial location $(x, y)$ and $\sigma_{t}$ is the standard deviation corresponding to the object size. The training objective is a penalty-reduced pixel-wise logistic regression with focal loss [16]:

$$
L_{c}=-\sum_{x y}\left\{\begin{array}{cc}
\left(1-\hat{C}_{x y}\right)^{\alpha} \log \left(\hat{C}_{x y}\right) & \text { if } C_{x y}=1 \\
\left(1-C_{x y}\right)^{\beta}\left(\hat{C}_{x y}\right)^{\alpha} & \text { otherwise } \\
\log \left(1-\hat{C}_{x y}\right) &
\end{array}\right.
$$

where $\alpha$ and $\beta$ are hyper-parameters of the focal loss. We empirically set $\alpha$ to 2 , and $\beta$ to 4 in our experiments.

\subsection{Size and Offset Regression}

As shown in Figure 2, the module contains two parallel branches. The size regression branch predicts the $\hat{W} \in \mathbb{R}^{\frac{H}{d} \times \frac{W}{d}}$ and $\hat{H} \in \mathbb{R}^{\frac{H}{d} \times \frac{W}{d}}$ while the offset regression branch estimates $\hat{\Delta} x \in \mathbb{R}^{\frac{H}{d} \times \frac{W}{d}}$ and $\hat{\Delta} y \in \mathbb{R}^{\frac{H}{d} \times \frac{W}{d}}$. The regressed size and offset maps are pixel-wisely corresponded to the estimated center points heatmap $\hat{C}$.

Both branches take the visual feature $E_{I}^{1}$ as input. The regression is conducted without using any expression features. The reason is that the spatial structure information is important for the regression, adding expression features may destroy the rich spatial information in the visual features. Both size and offset regression branches contain a $3 \times 3$ convolutional layer with ReLU followed by a $1 \times 1$ convolutional layer.

$L 1$ loss function is used during training. The object size loss $L_{\text {size }}$ and the local offset regression loss $L_{\text {off }}$ are defined as:

$$
\begin{array}{r}
L_{\text {size }}=\left|\hat{W}_{x^{g} y^{g}}-w^{g}\right|+\left|\hat{H}_{x^{g} y^{g}}-h^{g}\right| \\
L_{o f f}=\left|\hat{\Delta} x_{x^{g} y^{g}}-\delta x^{g}\right|+\left|\hat{\Delta} y_{x^{g} y^{g}}-\delta y^{g}\right|,
\end{array}
$$

where $w^{g}$ and $h^{g}$ are the ground truth width and height of the target box and $\delta x^{g}=\left(\frac{x^{g}}{d}-x^{g}\right)$ and $\delta y^{g}=\left(\frac{y_{g}}{d}-y^{g}\right)$ are the ground truth offset vector. $\hat{W}_{x^{g} y^{g}}$ is the value of $\hat{W}$ at the spatial location $\left(x^{g}, y^{g}\right)$ while $\hat{H}_{x^{g}} y^{g}, \hat{\Delta} x_{x^{g}} y^{g}$ and $\hat{\Delta} y_{x^{g} y^{g}}$ are defined similarly. Note that the regression loss acts only at the location of the center point $\left(x^{g}, y^{g}\right)$, all other locations are ignored.

\subsection{Loss and Inference}

The final loss is the weighted summation of three loss terms:

$$
\text { Loss }=L_{c}+\lambda_{\text {size }} L_{s i z e}+\lambda_{o f f} L_{o f f}
$$


where we set $\lambda_{\text {size }}$ to 0.1 and $\lambda_{\text {off }}$ to $1 . \lambda_{\text {size }}$ is equivalent to a normalized coefficient for the object size.

During inference, we select the point $\left(x_{t}, y_{t}\right)$ with the highest confidence score in the heatmap $\hat{C}$ as the target center point. The target size and offset are obtained from the corresponding position in the $\hat{W}, \hat{H}, \hat{\Delta x}$ and $\hat{\Delta y}$ as $\hat{W}_{x_{t}, y_{t}}$, $\hat{H}_{x_{t}, y_{t}}, \hat{\Delta} x_{x_{t}, y_{t}}$ and $\hat{\Delta} y_{x_{t}, y_{t}}$. The coordinates of the topleft and bottom-right corner of the target box are obtained by:

$$
\begin{aligned}
& \left(x_{t}+\hat{\Delta} x_{x_{t}, y_{t}}-\frac{\hat{W}_{x_{t}, y_{t}}}{2}, y_{t}+\hat{\Delta} y_{x_{t}, y_{t}}-\frac{\hat{H}_{x_{t}, y_{t}}}{2},\right. \\
& \left.x_{t}+\hat{\Delta} x_{x_{t}, y_{t}}+\frac{\hat{W}_{x_{t}, y_{t}}}{2}, y_{t}+\hat{\Delta} y_{x_{t}, y_{t}}+\frac{\hat{H}_{x_{t}, y_{t}}}{2}\right) .
\end{aligned}
$$

\section{Experiments}

In this section, we first introduce the experimental setting and implementation details, and then evaluate our method on four public benchmarks comparing to the state-of-the-art methods. After that, we analyze in detail the effectiveness of each component in our framework through a set of ablation experiments. Finally, we conduct an efficiency analysis followed by the qualitative results analysis.

\subsection{Experimental Setting}

Dataset. The experiments are conducted and evaluated on four common referring expression benchmarks, including RefClef [11], RefCOCO [11], RefCOCO+ [11] and RefCOCOg [20]. RefClef is also known as Refitgame, and is a subset of the ImageCLEF dataset. The other three datasets are all built on MS COCO images. RefCOCO and RefCOCO+ are collected in an interactive game, where the referring expressions tend to be short phrases. Comparing to RefCOCO, RefCOCO+ forbids using absolute location words and takes more attention on appearance description. To produce longer expressions, RefCOCOg is collected in a non-interactive setting. RefClef has 130, 363 expressions for 99, 296 objects in 19, 997 images. RefCOCO has 142,210 expressions for 50, 000 objects in 19, 994 images, RefCOCO+ has 141, 565 expressions for 49,856 objects in 19, 992 images, and RefCOCOg has 104, 560 expressions for 54,822 objects in 26,711 images.

Both RefCOCO and RefCOCO+ are divided into four subsets: 'train', 'val', 'testA' and 'testB'. The focus of the 'testA' and 'testB' are different. An image contains multiple people in 'testA' and multiple objects in 'testB'. For RefCOCOg, we follow the split in [32]. For fair comparison, we used the split released by [35] for RefClef.

Evaluation Metric. Following the detection proposal setting in the previous works, we use the Prec@0.5 to evaluate our method, where a predicted region is correct if its intersection over union (IOU) with the ground-truth bounding box is greater than 0.5 .

\begin{tabular}{|c|c|c|c|}
\hline & $\begin{array}{c}\text { Params } \\
\text { (Million) }\end{array}$ & $\begin{array}{c}\text { FLOPs } \\
\text { (Billion) }\end{array}$ & $\begin{array}{c}\text { Top-1 Error } \\
(\%)\end{array}$ \\
\hline VGG16 & 138 & 15.3 & 28.07 \\
ResNet-101 & 44.5 & 7.6 & 21.75 \\
DLA-34 & 18.4 & 3.5 & 25.32 \\
\hline
\end{tabular}

Table 1. The parameters, computation and top-1 error on ImageNet validation of the three backbone networks used in referring expression comprehension methods.

\subsection{Implementation Details}

We set hyper-parameters following Centernet [36]. Our RCCF method is also robust to these hyper-parameters. All experiments are conducted on the Titan Xp GPU and CUDA 9.0 with Intel Xeon CPU E5-2680v4@2.4G.

The resolution of the input image is $512 \times 512$, and we set the output stride to 4 . Thereby the output resolution is $128 \times 128$. Our proposed model is trained with Adam [12]. We train on 8 GPUs with a batch-size of 128 for 80 epochs, with a learning rate of $5 \mathrm{e}-4$ which is decreased by 10 at the 60 epochs, and again at 70 epochs. We use random shift and random scaling as the data augmentation. There is none augmentation during inference. The visual encoder are initialized with the weights pretrained on COCOs training images excluding the val/test set of RefCOCO series datasets, and the language encoder and the output heads are randomly initialized. For ablation study, we also conduct experiments on the visual encoder initialized with ImageNet [5] pretrain.

\begin{tabular}{c|c}
\hline Method & Precise @ 0.5 (\%) \\
\hline SCRC [10] & 17.93 \\
GroundR [25] & 26.93 \\
MCB [3] & 26.54 \\
CMN [9] & 28.33 \\
VC [35] & 31.13 \\
GGRE [19] & 31.85 \\
MNN [3] & 32.21 \\
CITE [23] & 34.13 \\
IGOP [30] & 34.70 \\
\hline Ours & $\mathbf{6 3 . 7 9}$
\end{tabular}

Table 2. Comparison with the state-of-the-arts on RefClef.

\subsection{Comparison to the State-of-the-art}

We compare RCCF to the state-of-the-art methods on four public benchmarks. The comparison results on RefClef dataset is shown in Table 2 while the results on the other three dataset are illustrated in Table 3 The previous methods use a 16-layer VGGNet [26] or a 101-layer ResNet [7] as the image encoder, while our proposed RCCF adopts DLA-34 [31] to encode images. The reason is that the VGG16 and ResNet-101 are not suitable for the key- 
point estimation alike tasks according to [15, 6] .

For fair comparison, we compare the two backbone networks with DLA-34 from three aspects in Table 1 . We can see the DLA-34 has the minimum parameters and computations (FLOPs), and its performance in image classification on ImageNet [5] is worse than ResNet-101.

Therefore, the performance gain of our RCCF comes from the framework itself, instead of more parameters or more complex backbone network. The baselines we compared with mainly use Faster-Rcnn [24], pretrained in object detection dataset, i.e., COCO and Visual Genome, to generate object proposals first, then matches the expression with all object proposals.

RefClef. The results in RefClef are presented in Table 2 Comparing to the state-of-the-art methods in RefClef, our method increases the state-of-the-arts by a significant margin from $34.70 \%$ to $63.79 \%$, almost double the precision.

RefCOCO, RefCOCO+ and RefCOCOg. Refer to Table 3 , our method outperforms existing methods in all evaluation sets on RefCOCO and RefCOCO+, and achieves comparable performance with the state-of-the-art method on RefCOCOg. Our result is a slightly inferior to MAttNet [32] in the RefCOCOg dataset. The performance gain of MAttNet partly comes from the additional supervision, such as attributes and class labels of region proposals, while our method only utilizes the language-image pair. Additionally, MAttNet uses a more complex backbone ResNet-101 while we only use DLA-34.

In conclusion, our method can achieve pretty-well performance in all of the four datasets. In addition, the twostage methods achieve much higher precision in the three RefCOCO series datasets than in RefClef. It is owing that all three RefCOCO series datasets are subsets of COCO, so the two-stage methods can train a very accurate detector based COCO object detection dataset, while RefClef does not have a such large corresponding object detection dataset. Therefore, traditional two-stage methods are heavily dependent on the object detector performance and the object detection dataset, while our novel RCCF framework avoid the explicit object detection stage and tackles the referring expression problem straightly.

\subsection{Ablation Studies}

In this section, we perform ablation studies from five different aspects on RefCOCO dataset to analyse the rationality and effectiveness of the proposed components in RCCF. The results are shown in Table 4

Fusion Strategy. In the first two rows, we report the results on two different fusion manners for the output correlation maps. In the first manner, we fuse the correlation by pixel-wisely taking the maximum value. To accomplish it, we concatenate the three output correlation maps, and obtain pixel-wise maximum across all channels. In the second manner, we generate the output heatmap by concatenating the three correlation maps, followed by a $1 \times 1$ convolutional layer. The results can be seen in the first row and the second row in Table 4. We conclude both the maximum fusion and concatenation are not as good as the average fusion shown in row 10 .

Filter Kernel Setting Here we perform ablation studies on the different variations of language filters (kernels). $3 \times 3$ Filter (row 3 ) is the method by expanding the language filter channels by 9 times, and reshaping it into $3 \times 3$. Then, we perform correlation filter using the $3 \times 3$ kernels. The result is almost the same with the 'Ours' with the $1 \times 1$ kernel (row 10). Considering the additional computational cost, we choose to use $1 \times 1$ kernel.

In row 4, we only generate one filter from the language feature, and perform correlation filtering on the three level visual features with the same kernel. In this case, the precision has dropped about 3 points. This shows that the diversity of the language kernels is important to match the visual features of different levels.

Single Level Visual Feature. In row 5, we perform the correlation filtering only based on the last level of the visual feature $E_{I}^{1}$ with single language kernel. The performance has dropped a lot from "Ours", but only dropped a little from the single language filter, multi-level visual features setting in row 4. Therefore, it can be concluded that the different language filters are sensitive to the different level of visual features.

Language-guided Regression. To verify whether the feature filtered by the language filter is suitable for the regression, we feed the concatenated feature of the three correlation maps into two convolutional layers in two regression branches. As shown in row 6, the performance drops a lot, about 6 points. Therefore, it is not a good choice to use language-guided features to regress the object size and offset in our RCCF framework.

Expression \& Image Encoder. The row 7 to row 9 of Table 4 show our method with various encoders. In row 7 , to explore the effect of the visual encoder pretrain model on the performance, we initialize the DLA-34 with ImageNet pretrain instead of $\mathrm{COCO}$ object detection pretrain. The results have dropped about 2 points, but also achieved comparable results to the state-of-the-art method. It proves that our method can also work well without any prior knowledge from object detection. In row 8, we use GloVe [22] as the word embedding. There is little change in the performance, so our method is robust to the two different language embeddings. In row 9, we replace the visual encoder with 


\begin{tabular}{|c|c|c|c|c|c|c|c|c|}
\hline & & & \multicolumn{2}{|c|}{ RefCOCO } & \multicolumn{2}{l|}{ RefCOCO+ } & RefCOCOg & \\
\hline & Method & Visual Encoder & testA & testB & testA & testB & test & Time (ms) \\
\hline 1 & MMI [20] & VGG16 & 64.90 & 54.51 & 54.03 & 42.81 & - & - \\
2 & NegBag [21] & VGG16 & 58.60 & 56.40 & - & - & 49.50 & - \\
3 & CG [19] & VGG16 & 67.94 & 55.18 & 57.05 & 43.33 & - & - \\
4 & Attr [18] & VGG16 & 72.08 & 57.29 & 57.97 & 46.20 & - & - \\
5 & CMN [9] [3] & VGG16 & 71.03 & 65.77 & 54.32 & 47.76 & - & - \\
6 & Speaker [33] & VGG16 & 67.64 & 55.16 & 55.81 & 43.43 & - & - \\
7 & Speaker+Listener+Reinforcer [34] & VGG16 & 72.94 & 62.98 & 58.68 & 47.68 & - & 1235 \\
8 & Spearker+Listener+Reinforcer [34] & VGG16 & 72.88 & 63.43 & 60.43 & 48.74 & - & 1332 \\
9 & VC[35] & VGG16 & 73.33 & 67.44 & 58.40 & 53.18 & - & 383 \\
10 & ParallelAttn [37] & VGG16 & 75.31 & 65.52 & 61.34 & 50.86 & - & - \\
11 & LGRANs [27] & VGG16 & 76.6 & 66.4 & 64.0 & 53.4 & - & - \\
12 & DGA [29] & VGG16 & 78.42 & 65.53 & 69.07 & 51.99 & 63.28 & 330 \\
\hline 13 & Spearker+Listener+Reinforcer [34] & ResNet-101 & 73.71 & 64.96 & 60.74 & 48.80 & 59.63 & - \\
14 & Spearker+Listener+Reinforcer [34] & ResNet-101 & 73.10 & 64.85 & 60.04 & 49.56 & 59.21 & - \\
15 & MAttNet [32] & ResNet-101 & 80.43 & 69.28 & 70.26 & 56.00 & $\mathbf{6 7 . 0 1}$ & 314 \\
\hline 16 & Ours & DLA-34 & $\mathbf{8 1 . 0 6}$ & $\mathbf{7 1 . 8 5}$ & $\mathbf{7 0 . 3 5}$ & $\mathbf{5 6 . 3 2}$ & 65.73 & 25 \\
\hline
\end{tabular}

Table 3. Comparison with state-of-the-art approaches on RefCOCO, RefCOCO+ and RefCOCOg.

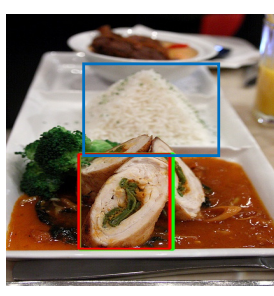

(a) "the middle piece of the chicken rollup"

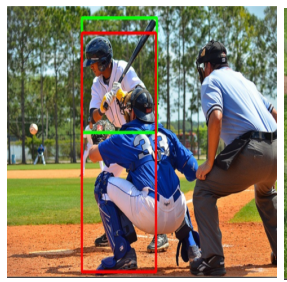

(g)"Baseball player holding the bat"

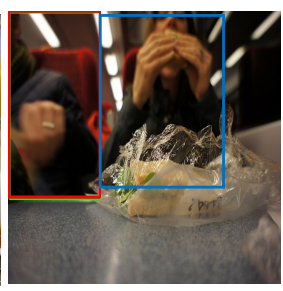

(b) "man's hand with ring on it"

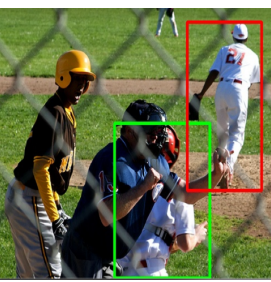

(h) " front guy in white"

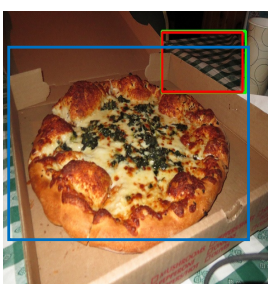

(c)" table behind box"

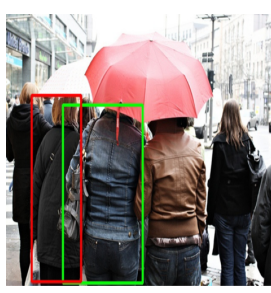

(i)" woman under umbrella left"

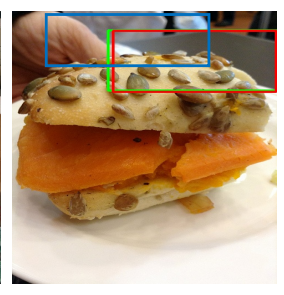

d)" The corner of the gray table visible to the right of the hand"

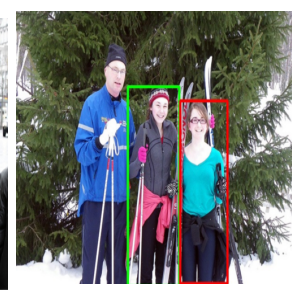

(j)“ woman"

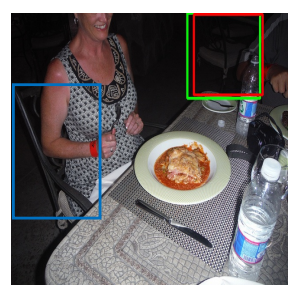

A steel chair near a lady
and back of the man"

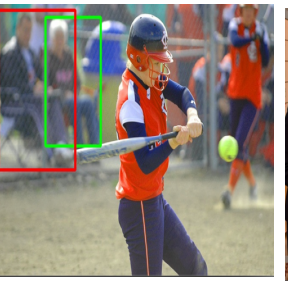

(k)" person behind fence on (I) the left white hair"

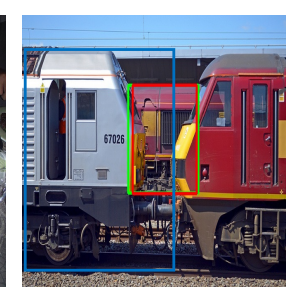

(f)"space between two train cars"

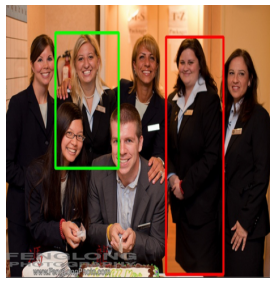

girl sitting with glasses"

Figure 3. Visualization results on RefCOCO series dataset. The first row (a-f) shows the comparisons of our approach with the state-ofthe-art method MAttNet. The second row shows some representative failure cases of our method. The red bounding-box represents the prediction of our method, the blue bounding-box represents the prediction of MAttNet, and the green bounding-box is the corresponding ground-truth.

a deeper network Hourglass-104 [15] in a single level setting. Comparing to the row 5, this setting has just improved a little, but this setting is much slower than our basic setting with DLA-34 during inference and training. More than 100 hours are needed for training and the inference speed is much lower.

\subsection{Efficiency Analysis}

Inference. As can be seen in Figure 1, our model runs at $25 \mathrm{~ms}$ per image on a single Titan Xp GPU and is the only real-time method in referring expression comprehen- sion area. In comparison, our method is 12 times faster than the state-of-the-art two-stage method MAttNet which needs to cost $314 \mathrm{~ms}$ for an image. For more detail comparison, the inference time per image of the first stage and the second stage of MAttNet are $262 \mathrm{~ms}$ and $52 \mathrm{~ms}$ respectively. The cost of either stage is longer than the total inference time of our method. More comparisons of the timing and precision can be found in Figure 1 .

Training. Our method is also fast to train. Training with DLA-34 on RefCOCO takes 35 hours in our synchronized 8-GPU implementation (1.78s per 128 image- 


\begin{tabular}{|c|c|c|c|c|}
\hline & & \multicolumn{2}{|c|}{ RefCOCO } & \\
\hline & Method & testA & testB & Time(ms) \\
\hline 1 & Maximum Fusion & 77.16 & 69.15 & 25 \\
2 & Concatenation & 79.85 & 69.83 & 26 \\
\hline 3 & 3x3 Filter & 80.83 & $\mathbf{7 2 . 0 1}$ & 26 \\
4 & Single Language Filter & 77.66 & 68.87 & 24 \\
\hline 5 & Single Level Visual Feature & 77.14 & 68.50 & 23 \\
\hline 6 & Language-guided Regression & 75.13 & 66.16 & 24 \\
\hline 7 & ImageNet Pretrained & 78.93 & 66.73 & 25 \\
8 & Glove Expression Encoder & 81.05 & 71.17 & 25 \\
9 & Hourglass Image Encoder & 78.12 & 69.38 & 80 \\
\hline 10 & Ours & $\mathbf{8 1 . 0 6}$ & 71.85 & 25 \\
\hline
\end{tabular}

Table 4. Ablation experiments on RefCOCO dataset.

language pairs mini-batch).

\subsection{Qualitative Results Analyses}

Correlation Map. Figure 4 shows the correlation map of the object center. We can see that given different expressions for the same image, the correlation map responses to different locations. Otherwise, it can be seen that the response is very high in areas near the center of object described by the expression. Moreover, there are very small responses in other locations. It shows that our model is capable to well match the expression and visual features.

Comparison to the State-of-the-art. In the first row of Figure 3, we compare our method with the state-of-the-art method MAttNet. Our method can accurately localize the target objects under the guidance of the language, even if the objects are hard to be detected for common object detectors. For example, although the described objects "piece" (Figure 3(a)) and "space" (Figure 3(f)) are very abstract and not included in the COCO categories, our method can still find them through the expression. It proves that our method can well match expression and visual features. While MAttNet is dependent on the object detector, MAttNet will fail if the object category is beyond the scope of the detector category set.

Failure Case Analysis. The second row of Figure 3 illustrates some possible failure cases. As shown in the Figure $3(\mathrm{~g})$, we find the right object, but fail to accurately locate the bounding-box. Another example is shown in Figure $3 \mathrm{~h}$ ), the target object is occluded heavily, and the model cannot capture enough appearance information. In addition, the ground-truth error may occur. For example in Figure $3(\mathrm{j})$, there are more than one target objects described by the expression. Some failure cases may be caused by that target object lies in the background and it is difficult to find the appearance feature described by the expression. In addition, when expression is very complex and long, our model may fail to understand it well, such as the case in Figure $3(1)$. We leave how to solve these failure cases as interesting future works.

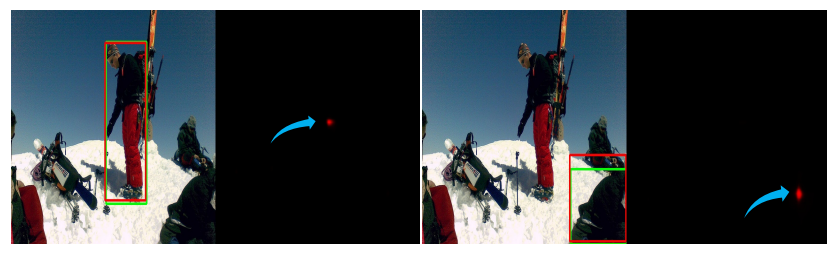

"guy with red pants standing"

"right bottom partial black"

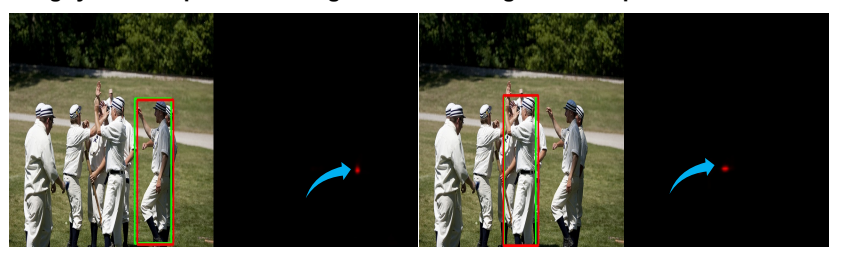

"guy all the way right in front"

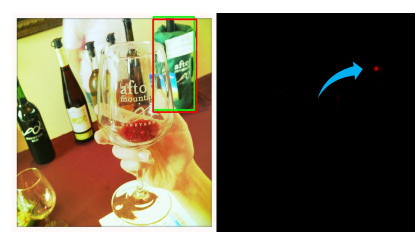

"the green cup on the top right has the word after on it" "guy in the center most to the front"

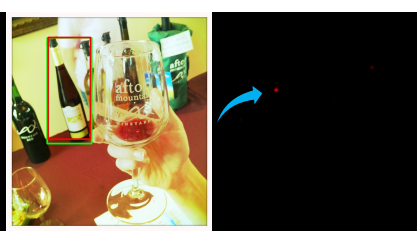

"tall bottle with yellow tag"
Figure 4. Visualization of visual grounding results and correlation map. On the left image, the red bounding-box represents the prediction of our method while the green bounding-box represents the ground-truth. The right image shows the corresponding predicted correlation map for the center point of the object (pointed by the blue arrow).

\section{Conclusion and Future Works}

In this paper, we propose a real-time and highperformance framework for referring expression comprehension. Completely different from the previous two-stage methods, our proposed RCCF directly localizes the object given an expression by predicting the object center through computing a correlation map between the referent and the image. The RCCF is able to achieve state-of-the-art performance in four referring expression datasets at real-time speed. For future work, on the one hand, we plan to explore how to capture more context information from expression and image, and thus understand the expression better. On the other hand, the referring expression is difficult to annotate, so we want to explore how to utilize other easy annotated types of datasets to train our method, like object detection, image caption.

Acknowledgement This work was partially supported by the State Key Development Program (Grant 2016YFB1001004), Sensetime Ltd. Group, the National Natural Science Foundation of China ( Grant 61876177, Grant 61976250), Beijing Natural Science Foundation (L182013, 4202034), Zhejiang Lab (No. 2019KD0AB04 ), and Fundamental Research Funds for the Central Universities. 


\section{References}

[1] Luca Bertinetto, Jack Valmadre, Joao F Henriques, Andrea Vedaldi, and Philip HS Torr. Fully-convolutional siamese networks for object tracking. In ECCV, 2016.

[2] David S Bolme, J Ross Beveridge, Bruce A Draper, and Yui Man Lui. Visual object tracking using adaptive correlation filters. In $C V P R, 2010$.

[3] Kan Chen, Rama Kovvuri, Jiyang Gao, and Ram Nevatia. Msrc: Multimodal spatial regression with semantic context for phrase grounding. In ICMR, 2017.

[4] Jifeng Dai, Haozhi Qi, Yuwen Xiong, Yi Li, Guodong Zhang, Han $\mathrm{Hu}$, and Yichen Wei. Deformable convolutional networks. In ICCV, 2017.

[5] Jia Deng, Wei Dong, Richard Socher, Li-Jia Li, Kai Li, and Li Fei-Fei. Imagenet: A large-scale hierarchical image database. In $C V P R, 2009$.

[6] Kaiwen Duan, Song Bai, Lingxi Xie, Honggang Qi, Qingming Huang, and Qi Tian. Centernet: Object detection with keypoint triplets. arXiv preprint arXiv:1904.08189, 2019.

[7] Kaiming He, Xiangyu Zhang, Shaoqing Ren, and Jian Sun. Deep residual learning for image recognition. In $C V P R$, 2016.

[8] João F Henriques, Rui Caseiro, Pedro Martins, and Jorge Batista. High-speed tracking with kernelized correlation filters. TPAMI, 2014.

[9] Ronghang Hu, Marcus Rohrbach, Jacob Andreas, Trevor Darrell, and Kate Saenko. Modeling relationships in referential expressions with compositional modular networks. In CVPR, 2017.

[10] Ronghang Hu, Huazhe Xu, Marcus Rohrbach, Jiashi Feng, Kate Saenko, and Trevor Darrell. Natural language object retrieval. In $C V P R, 2016$.

[11] Sahar Kazemzadeh, Vicente Ordonez, Mark Matten, and Tamara Berg. Referitgame: Referring to objects in photographs of natural scenes. In EMNLP, 2014.

[12] Diederik P Kingma and Jimmy Ba. Adam: A method for stochastic optimization. arXiv preprint arXiv:1412.6980, 2014.

[13] Ranjay Krishna, Yuke Zhu, Oliver Groth, Justin Johnson, Kenji Hata, Joshua Kravitz, Stephanie Chen, Yannis Kalantidis, Li-Jia Li, David A Shamma, et al. Visual genome: Connecting language and vision using crowdsourced dense image annotations. IJCV, 2017.

[14] Alex Krizhevsky, Ilya Sutskever, and Geoffrey E Hinton. Imagenet classification with deep convolutional neural networks. In NIPS, 2012.

[15] Hei Law and Jia Deng. Cornernet: Detecting objects as paired keypoints. In ECCV, 2018.

[16] Tsung-Yi Lin, Priya Goyal, Ross Girshick, Kaiming He, and Piotr Dollár. Focal loss for dense object detection. In ICCV, 2017.

[17] Tsung-Yi Lin, Michael Maire, Serge Belongie, James Hays, Pietro Perona, Deva Ramanan, Piotr Dollár, and C Lawrence Zitnick. Microsoft coco: Common objects in context. In ECCV, 2014.
[18] Jingyu Liu, Liang Wang, and Ming-Hsuan Yang. Referring expression generation and comprehension via attributes. In ICCV, 2017.

[19] Ruotian Luo and Gregory Shakhnarovich. Comprehensionguided referring expressions. In CVPR, 2017.

[20] Junhua Mao, Jonathan Huang, Alexander Toshev, Oana Camburu, Alan L Yuille, and Kevin Murphy. Generation and comprehension of unambiguous object descriptions. In CVPR, 2016.

[21] Varun K Nagaraja, Vlad I Morariu, and Larry S Davis. Modeling context between objects for referring expression understanding. In ECCV, 2016.

[22] Jeffrey Pennington, Richard Socher, and Christopher Manning. Glove: Global vectors for word representation. In EMNLP, 2014.

[23] Bryan A Plummer, Paige Kordas, M Hadi Kiapour, Shuai Zheng, Robinson Piramuthu, and Svetlana Lazebnik. Conditional image-text embedding networks. In ECCV, 2018.

[24] Shaoqing Ren, Kaiming He, Ross Girshick, and Jian Sun. Faster r-cnn: Towards real-time object detection with region proposal networks. In NIPS, 2015.

[25] Anna Rohrbach, Marcus Rohrbach, Ronghang Hu, Trevor Darrell, and Bernt Schiele. Grounding of textual phrases in images by reconstruction. In ECCV, 2016.

[26] Karen Simonyan and Andrew Zisserman. Very deep convolutional networks for large-scale image recognition. arXiv preprint arXiv:1409.1556, 2014.

[27] Peng Wang, Qi Wu, Jiewei Cao, Chunhua Shen, Lianli Gao, and Anton van den Hengel. Neighbourhood watch: Referring expression comprehension via language-guided graph attention networks. In CVPR, 2019.

[28] Sibei Yang, Guanbin Li, and Yizhou Yu. Cross-modal relationship inference for grounding referring expressions. In CVPR, 2019.

[29] Sibei Yang, Guanbin Li, and Yizhou Yu. Dynamic graph attention for referring expression comprehension. In ICCV, 2019.

[30] Raymond Yeh, Jinjun Xiong, Wen-Mei Hwu, Minh Do, and Alexander Schwing. Interpretable and globally optimal prediction for textual grounding using image concepts. In NIPS, 2017.

[31] Fisher Yu, Dequan Wang, Evan Shelhamer, and Trevor Darrell. Deep layer aggregation. In CVPR, 2018.

[32] Licheng Yu, Zhe Lin, Xiaohui Shen, Jimei Yang, Xin Lu, Mohit Bansal, and Tamara L Berg. Mattnet: Modular attention network for referring expression comprehension. In CVPR, 2018.

[33] Licheng Yu, Patrick Poirson, Shan Yang, Alexander C Berg, and Tamara L Berg. Modeling context in referring expressions. In ECCV, 2016.

[34] Licheng Yu, Hao Tan, Mohit Bansal, and Tamara L Berg. A joint speaker-listener-reinforcer model for referring expressions. In CVPR, 2017.

[35] Hanwang Zhang, Yulei Niu, and Shih-Fu Chang. Grounding referring expressions in images by variational context. In CVPR, 2018. 
[36] Xingyi Zhou, Dequan Wang, and Philipp Krähenbühl. Objects as points. arXiv preprint arXiv:1904.07850, 2019.

[37] Bohan Zhuang, Qi Wu, Chunhua Shen, Ian Reid, and Anton van den Hengel. Parallel attention: A unified framework for visual object discovery through dialogs and queries. In CVPR, 2018. 